\title{
Eine quantitative Perspektive auf die Priorität von Risikofaktoren in IT-Projekten
}

\author{
Stefan Hörmann, Michael Schermann, Helmut Kromar \\ Lehrstubl für Wirtschaftsinformatik, \\ Technische Universität München
}

\section{Einleitung}

Sowohl die Praxis als auch die Wissenschaft argumentiert, dass Risikomanagement essenziell für den Erfolg von IT Projekten ist (Schmidt et al. 2001; Wallace et al. 2004). Risikomanagement ermöglicht Projektmanagern Risiken und deren zu Grunde liegenden Risikofaktoren zu identifizieren, zu analysieren, zu kontrollieren und zu überwachen (Chapman und Ward 1996). Es liegt auf der Hand, dass die Fähigkeit eines Projektmanagers, die für ein Projekt wichtigen Risiken und Risikofaktoren zu identifizieren, die Effektivität des Projektrisikomanagement maßgeblich beeinflusst.

Aus diesem Grund fokussiert ein beträchtlicher Teil der ITProjektrisikoforschung darauf, eine Rangfolge von Risiken und Risikofaktoren bzgl. deren Bedeutung für den Projekterfolg zu ermitteln (Barki et al. 1993; Kappelman et al. 2006; Schmidt et al. 2001). Obwohl diese Ansätze nahezu keine Erklärungen für ihre Priorisierung von Risikofaktoren liefern, kommen sie weitestgehend überein, dass personen- und prozessbezogene Risikofaktoren die bedeutendste Rolle in IT-Projekten spielen, wohingegen technikbezogene Risikofaktoren vernachlässigbar sind (Kappelman et al. 2006; Schmidt et al. 2001).

Die meisten der Rangfolgen basieren auf der Erfahrung von Projektmanagern, d.h. Projektmanager wurden aufgefordert, Risikofaktoren $\mathrm{zu}$ gewichten. Risikorangfolgen, die auf anderen Datenquellen basieren, existieren nach Wissen der Autoren nicht. Zudem ist der Großteil der bestehenden Studien beschreibender Natur. Auch hier existieren unserer Ansicht nach kaum Erklärungsansätze bzgl. Struktur und Reihenfolge der Risikolisten.

Das Anliegen dieses Beitrags ist es, bestehende Forschung kritisch zu hinterfragen, indem wir die relative Bedeutung von Risikofaktoren quantitativ untersuchen. Unsere Forschungsfrage lautet: Welche Mechanismen liegen Rangfolgen von Risikofaktoren in IT-Projekten zu Grunde? Dazu analysieren wir das Risikodatenarchiv 
von ALPHA ${ }^{1}$, einem großen, multinationalen Softwareunternehmen. Die Risikoberichte des Datenarchivs dienen dazu, Projektvorschläge zu bewerten, den Status des Projekts aus Unternehmensperspektive zu betrachten und kritische Projektsituationen frühzeitig zu signalisieren. Wir konsolidieren die einzelnen Risikoberichte in einer Risikodatenbank, um eine Rangfolge zu erstellen.

Der Beitrag ist wie folgt strukturiert: Im nächsten Abschnitt untersuchen wir bestehende Vorschläge für Rangfolgen von Risikofaktoren in IT-Projekten. Wir zeigen, dass die meisten Studien nur eine beschreibende Perspektive auf die relative Bedeutung von Risikofaktoren geben. Im darauffolgenden Abschnitt stellen wir die Ergebnisse unserer Analyse vor und ziehen Vergleiche $\mathrm{zu}$ bestehenden Rangfolgen. Unsere Ergebnisse deuten darauf hin, dass Projektmanager technikbezogene Risikofaktoren als sehr bedeutend für den Projekterfolg einstufen. Um erste Erklärungsansätze für diese Diskrepanz zu geben, wenden wir Theorien aus den Gebieten Risikomanagement und Verhaltensforschung an. Der Beitrag schließt mit einer kritischen Zusammenfassung und einem Ausblick.

\section{Stand der Literatur}

Alter und Ginzberg (1978) untersuchten in einer der ersten Studien zu Risiken in IT-Projekten den Softwareentwicklungsprozess und ordneten den Prozessschritten insgesamt acht Risikofaktoren zu. Zmud (1980) argumentierte, dass große Softwareentwicklungsprojekte hauptsächlich Managementprobleme hervorrufen und technische Probleme nur eine untergeordnete Rolle spielen. Zmud (1980) führt jedoch aus, dass Managementprobleme oftmals ihre Ursachen in technischen Problemen haben. McFarlan (1981) stellt einen Risiko-Portfolioansatz vor und hob die Bedeutung einer unternehmensweiten Risikoperspektive hervor. Böhm (1991) verdeutlicht die Bedeutung des Risikomanagements für das Projektmanagement. Er unterstreicht dabei die Risikopriorisierung nach der Höhe der jeweiligen Risikoexposition, die er anhand der Multiplikation von Eintrittswahrscheinlichkeit und Auswirkung des Risikofaktors berechnet. Tiwana und Keil (2004) führen die drei wesentlichen Risikobereiche „Projektmanagement“, „Soziales Teilsystem“ und „Technisches Teilsystem“ eines IT-Projekts ein. Tabelle 1 zeigt eine Auswahl an Studien, die sich unter anderem nach der gewählten Risikoperspektive oder dem Abstraktionslevel unterscheiden lassen.

Aufgrund eines ähnlichen Abstraktionsgrades werden im Folgenden die Studien von Schmidt et al. (2001) und Kappelman et al. (2006) als Vergleichsobjekte herangezogen. Der Großteil der von Schmidt et al. (2001) identifizierten 53 Risikofaktoren stammt dabei aus den Risikodomänen „Projektmanagement” und „Soziales Teilsystem". Nur zwei der identifizierten Risikofaktoren beziehen sich auf die Domäne „Technisches Teilsystem”.

${ }^{1}$ Name geändert. 
Tabelle 1: Vergleich von Studien zu Risikofaktoren (Auswahl)

\begin{tabular}{|c|c|c|c|c|c|c|c|}
\hline Studie & 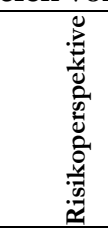 & 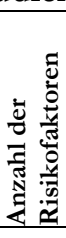 & 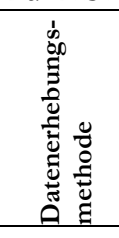 & 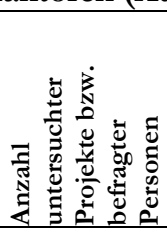 & 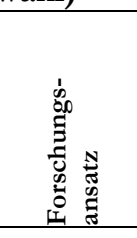 & 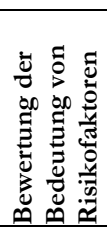 & 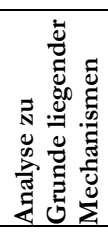 \\
\hline $\begin{array}{l}\text { Barki et al. } \\
\text { (1993) }\end{array}$ & $\begin{array}{l}\text { Unter- } \\
\text { nehmen }\end{array}$ & 34 & Umfrage & 120 & $\begin{array}{l}\text { Empirisch- } \\
\text { Quantitativ }\end{array}$ & Nein & Nein \\
\hline $\begin{array}{l}\text { Moynihan } \\
\text { (1997) }\end{array}$ & Projekt & 22 & Umfrage & 42 & $\begin{array}{l}\text { Empirisch- } \\
\text { Qualitativ }\end{array}$ & Nein & Nein \\
\hline $\begin{array}{l}\text { Jiang und Klein } \\
(2000)\end{array}$ & Projekt & 12 & Umfrage & 86 & $\begin{array}{l}\text { Empirisch- } \\
\text { Quantitativ }\end{array}$ & $\mathrm{Ja}$ & Nein \\
\hline $\begin{array}{l}\text { Schmidt et al. } \\
\text { (2001) }\end{array}$ & Projekt & 53 & $\begin{array}{l}\text { Delphi } \\
\text { Studie }\end{array}$ & 41 & $\begin{array}{l}\text { Empirisch- } \\
\text { Qualitativ }\end{array}$ & $\mathrm{Ja}$ & Nein \\
\hline $\begin{array}{l}\text { Wallace et al. } \\
\text { (2004) }\end{array}$ & Projekt & 6 & Umfrage & 507 & $\begin{array}{l}\text { Empirisch- } \\
\text { Quantitativ }\end{array}$ & Nein & Ja \\
\hline $\begin{array}{l}\text { Kappelman et al. } \\
(2006)\end{array}$ & $\begin{array}{l}\text { Projekt/ } \\
\text { Unter- } \\
\text { nehmen }\end{array}$ & 12 & Umfrage & 55 & $\begin{array}{l}\text { Empirisch- } \\
\text { Quantitativ }\end{array}$ & $\mathrm{Ja}$ & Nein \\
\hline
\end{tabular}

Die Autoren nehmen an, dass der niedrige Einfluss der technikbezogenen Risikofaktoren auf die bessere Performanz und Skalierbarkeit von Hard- und Software sowie die weitverbreitete Nutzung von graphischen Benutzeroberflächen zurückzuführen ist - eine Begründung die angesichts der steigenden technischen Komplexität etwas verwundert. Als Erklärungsansatz für die Reihenfolge der Risikofaktoren schlagen Schmidt et al. (2001) vor, dass Projektmanager die Risikofaktoren im Hinblick auf deren Kontrollierbarkeit bewerten. Eine mittlere Kontrollierbarkeit führt demnach zu einer hohe Bewertung durch Projektmanager (March und Shapira 1987). Ein hohes Maß an Kontrolle bzw. keine Kontrolle führen zu niedrigen Bewertungen oder gar zu einer Nicht-Beachtung der Risikofaktoren.

Ähnlich wie bereits bei Schmidt et al. (2001) wird keiner der zwölf wesentlichen Risikofaktoren nach Kappelman et al. (2006) dem technischen Teilsystem zugeordnet. Kappelman et al. (2006) legen hier dar, dass ihre Ergebnisse angesichts dessen, dass IT-Projekte so gut wie nie aufgrund technischer Probleme scheitern, nicht überraschend seien.

Insgesamt muss kritisch angemerkt werden, dass trotz der hohen praktischen Relevanz kaum Schlussfolgerungen bzgl. der relativen Bedeutung von Risikofaktoren getroffen werden. Diejenigen Studien, die eine Priorisierung von Risikofaktoren vornehmen, stimmen darin überein, dass Risikofaktoren aus den Domänen "Projektmanagement" und "Soziales Teilsystem" eine höhere Bedeutung haben, als Risiken, die dem technischen Teilsystem zuzuordnen sind. Erklärungen für diese Einschätzungen werden jedoch kaum geliefert. Ebenso ist die Datengrundlage nahezu aller Studien ausschließlich für Forschungszwecke gewonnen worden, 
d. h. über Umfragen oder Interviews. Wie einige der Autoren selbst bemerken, ist es wahrscheinlich, dass durch diese Form der Datenerhebung eine Verzerrung entsteht (Barki et al. 1993; Tiwana und Keil 2004). Der im Folgenden vorgestellte Weg, Archivdaten zu analysieren, vermeidet einen großen Teil dieser potenziellen Verzerrung sowie Probleme der Datenerhebung (Schmidt et al. 2001).

\section{Studie}

Unsere Analyse zielt darauf ab, eine Rangfolge von Risikofaktoren für IT-Projekte $\mathrm{zu}$ entwickeln. Unsere Priorisierung basiert auf den Risikobewertungen von Projektmanagern eines großen internationalen Softwareunternehmens (ALPHA), die zwischen 2004 und 2007 vorgenommen wurden.

\subsection{Datenerhebung}

Das Risikomanagement bei ALPHA orientiert sich an einem Standardprozess, der die vier Phasen "Risikoidentifikation", "Risikobewertung", "Risikomaßnahmenplanung" sowie "Risikoüberwachung" umfasst. Der Prozess läuft kontinuierlich vor und während des Projekts und wird durch den jeweiligen Projektmanager und teilweise durch das Projektteam durchgeführt. Je nach Wert des Projekts wird es dabei von einer zentralen Risikofunktion begleitet. Die Risikoidentifikation wird durch eine Risikocheckliste, die insgesamt 317 Fragen und 45 verschiedene Risikotypen enthält, unterstützt. Unter anderem werden die identifizierten Risikofaktoren nach Auswirkung (von 1, „unbedeutend“, bis 5, „katastrophal “) und Eintrittswahrscheinlichkeit bewertet. Nachdem Risikofaktoren identifiziert und bewertet wurden, findet die Maßnahmenplanung statt und die Risikoüberwachung beginnt.

Die Ergebnisse des gesamten Prozesses werden in einer oder mehreren Kalkulationstabellen gespeichert, von denen wir insgesamt 1548 zur Analyse heranziehen konnten. Davon war es uns möglich, 1222 auszuwerten. Die restlichen 326 Dateien waren entweder beschädigt, leer oder konnten keinem Kunden bzw. keinem Projekt zugeordnet werden. Wir lasen die Daten mittels eines Extraktionsprogramms aus und kontrollierten manuell nach, um eine hohe Datenqualität sicherzustellen.

\subsection{Datenanalyse}

Nachdem wir den Datensatz um Duplikate und unvollständige Einträge bereinigt hatten, verblieben 4570 Risikofaktoren aus 111 Projekten. Tabelle 2 zeigt verschiedene deskriptive Statistiken der drei Untersuchungsvariablen „Auswirkung“, „Eintrittswahrscheinlichkeit“ und „Risikoexposition“ (Auswirkung 
x Eintrittswahrscheinlichkeit). Wir treffen die Annahme, dass sich die Bedeutung eines Risikofaktors in der Variable „Risikoexposition“ widerspiegelt.

Tabelle 2: Deskriptive Statistiken der Untersuchungsvariablen

\begin{tabular}{|l|c|c|c|c|}
\hline Variablen & Mittelwert & Standardabweichung & Minimum & Maximum \\
\hline Auswirkung & 2,58 & 1,23 & 0,00 & 5,00 \\
\hline Eintrittswahrscheinlichkeit & 0,45 & 0,21 & 0,00 & 0,99 \\
\hline Risikoexposition & 1,22 & 0,86 & 0.00 & 4,95 \\
\hline
\end{tabular}

N: 4570

Basierend auf der mittleren Risikoexposition sind die zehn bedeutendsten der oben gelisteten Risikofaktoren in

Tabelle 3 genauer beschrieben. Insgesamt wurden 45 Risikofaktoren identifiziert, deren Beschreibung jedoch den Rahmen des Beitrags sprengen würde. Um einen Vergleich zu bestehenden Rangfolgen ziehen zu können, verknüpfen wir die Risikofaktoren mit den von Wallace et al. (2004) vorgeschlagenen Risikodomänen „Technisches Teilsystem“, „Soziales Teilsystem“ und „Projektmanagement“.

\section{Ergebnisse}

Tabelle 4 stellt die zehn bedeutendsten Risikofaktoren unserer Rangfolge mit denen der Rangfolgen von Schmidt et al. (2001) und Kappelman et al. (2006) gegenüber.

Wie zu erkennen, weichen die Risikobewertungen der Projektmanager von ALPHA erheblich von den Priorisierungen der anderen beiden Studien ab. Schmidt et al. (2001) und Kappelman et al. (2006) betrachten ausschließlich Risikofaktoren des Projektmanagements sowie des sozialen Teilsystems und sind bzgl. der Reihenfolge der Risiken vergleichbar. Beispielsweise erachten beide Studien die Unterstützung durch das Management sowie ein effektives Projektmanagement für wichtig. Dahingegen heben die Projektmanager bei ALPHA die Bedeutung von Risikofaktoren hervor, die dem technischen Teilsystem zugeordnet werden können, so wie z. B. eine unpassende technische Infrastruktur oder Abhängigkeiten zwischen zu entwickelnden Komponenten. Insgesamt lassen sich in unserer Priorisierung nur fünf der zehn bedeutendsten Risikofaktoren dem sozialen Teilsystem oder der Projektmanagementdomäne zuordnen. Außer dem Risikofaktor „Post Go Live Approach Not Defined“, der teilweise mit den Risikofaktoren „No Planning or Inadequate Planning“ bzw. „Ineffective Schedule Planning“ verglichen werden kann, sind die Priorisierungen gänzlich unterschiedlich. 
Tabelle 3: Risikoexposition je Faktor und Verknüpfung mit Risikodomänen

\begin{tabular}{|c|c|c|c|c|c|c|}
\hline شૈ & Z & 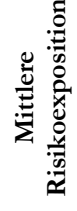 & 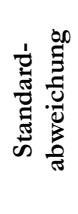 & Е్స్ & 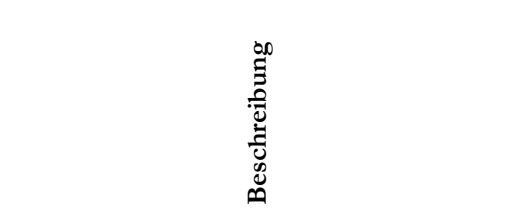 & 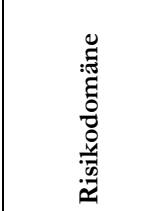 \\
\hline 1 & 49 & 1,93 & 1,24 & $\begin{array}{l}\text { Inadequate } \\
\text { Technical } \\
\text { Infrastructure }\end{array}$ & $\begin{array}{l}\text { The customer's expectations are not } \\
\text { consistent with the complexities of the } \\
\text { project. }\end{array}$ & $\begin{array}{l}\text { Technisches } \\
\text { Teilsystem }\end{array}$ \\
\hline 2 & 135 & 1,69 & 0,89 & $\begin{array}{l}\text { Customer } \\
\text { Expectations }\end{array}$ & $\begin{array}{l}\text { The Customer's expectations are not } \\
\text { consistent with the complexities of the } \\
\text { project. }\end{array}$ & $\begin{array}{l}\text { Soziales } \\
\text { Teilsystem }\end{array}$ \\
\hline 3 & 114 & 1,59 & 0,77 & $\begin{array}{l}\text { Core } \\
\text { Development } \\
\text { Dependencies }\end{array}$ & $\begin{array}{l}\text { Dependencies between ALPHA component } \\
\text { release planning and the development project } \\
\text { have not been considered or are unclear, or } \\
\text { the custom development project is based on } \\
\text { one or several unstable ALPHA components }\end{array}$ & $\begin{array}{l}\text { Technisches } \\
\text { Teilsystem }\end{array}$ \\
\hline 4 & 129 & 1,53 & 1,01 & $\begin{array}{l}\text { Complex System } \\
\text { Architecture }\end{array}$ & $\begin{array}{l}\text { A complex or state-of-the-art system } \\
\text { architecture is required to meet the } \\
\text { requirements (whether or not the Customer is } \\
\text { aware of or acknowledges the complexity). }\end{array}$ & $\begin{array}{l}\text { Technisches } \\
\text { Teilsystem }\end{array}$ \\
\hline 5 & 172 & 1,47 & 0,91 & $\begin{array}{l}\text { Post Go Live } \\
\text { Approach Not } \\
\text { Defined }\end{array}$ & $\begin{array}{l}\text { The approach and responsibilities for post } \\
\text { Go-Live Application or System Management } \\
\text { have not been determined }\end{array}$ & $\begin{array}{l}\text { Projektmanag } \\
\text { ement }\end{array}$ \\
\hline 6 & 40 & 1,42 & 0,99 & $\begin{array}{l}\text { Customer } \\
\text { Financial } \\
\text { Obligations }\end{array}$ & $\begin{array}{l}\text { The customer may be unable or unwilling to } \\
\text { meet its financial obligations under the } \\
\text { contract. }\end{array}$ & $\begin{array}{l}\text { Soziales } \\
\text { Teilsystem }\end{array}$ \\
\hline 7 & 204 & 1,37 & 0,91 & $\begin{array}{l}\text { Expected } \\
\text { Performance } \\
\text { Issues }\end{array}$ & $\begin{array}{l}\text { Performance issues are expected either due to } \\
\text { the high number of transactions, product } \\
\text { limitations, or volumes are unknown. }\end{array}$ & $\begin{array}{l}\text { Technisches } \\
\text { Teilsystem }\end{array}$ \\
\hline 8 & 203 & 1,36 & 0,87 & $\begin{array}{l}\text { Customer Inability } \\
\text { to Undertake } \\
\text { Project }\end{array}$ & $\begin{array}{l}\text { The customer does not have the ability, skills } \\
\text { and/or culture to successfully undertake the } \\
\text { project. }\end{array}$ & $\begin{array}{l}\text { Soziales } \\
\text { Teilsystem }\end{array}$ \\
\hline 9 & 242 & 1,35 & 0,98 & $\begin{array}{l}\text { Non-T\&M } \\
\text { Payment Terms }\end{array}$ & $\begin{array}{l}\text { The proposed services agreement is other } \\
\text { than Time and Materials and/or contains } \\
\text { non-standard prices, future price protection, } \\
\text { or non-standard payments terms. }\end{array}$ & $\begin{array}{l}\text { Soziales } \\
\text { Teilsystem }\end{array}$ \\
\hline 10 & 191 & 1,34 & 0,93 & $\begin{array}{l}\text { Functionality } \\
\text { Gaps }\end{array}$ & $\begin{array}{l}\text { There are gaps between the customer's } \\
\text { business requirements and ALPHA's } \\
\text { current/expected functionality }\end{array}$ & $\begin{array}{l}\text { Technisches } \\
\text { Teilsystem }\end{array}$ \\
\hline
\end{tabular}


Tabelle 4: Vergleich der Rangfolgen

\begin{tabular}{|c|c|c|c|c|c|c|}
\hline Rang & ALPHA & & Schmidt et al. (2001) & & Kappelman et al. (2006) & \\
\hline 1 & $\begin{array}{l}\text { Inadequate Technical } \\
\text { Infrastructure }\end{array}$ & $\mathrm{T}$ & $\begin{array}{l}\text { Lack of Effective Project } \\
\text { Management skills }\end{array}$ & $\mathrm{P}$ & $\begin{array}{l}\text { Lack of Top Management } \\
\text { Support }\end{array}$ & $\mathrm{S}$ \\
\hline 2 & $\begin{array}{l}\text { Customer } \\
\text { Expectations }\end{array}$ & S & $\begin{array}{l}\text { Lack of Top Management } \\
\text { commitment }\end{array}$ & S & $\begin{array}{l}\text { Lack of Documented } \\
\text { Requirements }\end{array}$ & $\mathrm{P}$ \\
\hline 3 & $\begin{array}{l}\text { Core Development } \\
\text { Dependencies }\end{array}$ & $\mathrm{T}$ & $\begin{array}{l}\text { Lack of Required Skills in } \\
\text { Project Personnel }\end{array}$ & $\mathrm{P}$ & Weak Project Manager & $\mathrm{P}$ \\
\hline 4 & $\begin{array}{l}\text { Complex System } \\
\text { Architecture }\end{array}$ & $\mathrm{T}$ & $\begin{array}{l}\text { Not Managing Change } \\
\text { Properly }\end{array}$ & $\mathrm{P}$ & $\begin{array}{l}\text { No Change Control Process } \\
\text { (Change Management) }\end{array}$ & $\mathrm{P}$ \\
\hline 5 & $\begin{array}{l}\text { Post Go Live } \\
\text { Approach Not } \\
\text { Defined }\end{array}$ & $\mathrm{P}$ & $\begin{array}{l}\text { No Planning or } \\
\text { Inadequate Planning }\end{array}$ & $\mathrm{P}$ & $\begin{array}{l}\text { No Stakeholder Involvement } \\
\text { and/or Participation }\end{array}$ & S \\
\hline 6 & $\begin{array}{l}\text { Customer Financial } \\
\text { Obligations }\end{array}$ & $\mathrm{S}$ & $\begin{array}{l}\text { Misunderstanding the } \\
\text { Requirements }\end{array}$ & $\mathrm{P}$ & $\begin{array}{l}\text { Ineffective Schedule Planning } \\
\text { and/or Management }\end{array}$ & $\mathrm{P}$ \\
\hline 7 & $\begin{array}{l}\text { Expected Performance } \\
\text { Issues }\end{array}$ & $\mathrm{T}$ & Artificial Deadlines & $\mathrm{P}$ & $\begin{array}{l}\text { Weak Commitment of Project } \\
\text { Team }\end{array}$ & $\mathrm{P}$ \\
\hline 8 & \begin{tabular}{|l|} 
Customer Inability to \\
Undertake Project \\
\end{tabular} & $S$ & $\begin{array}{l}\text { Failure to Gain User } \\
\text { Commitment }\end{array}$ & S & $\begin{array}{l}\text { Communication Breakdown } \\
\text { among Stakeholders }\end{array}$ & S \\
\hline 9 & $\begin{array}{l}\text { Non-T\&M Payment } \\
\text { Terms }\end{array}$ & $\mathrm{S}$ & $\begin{array}{l}\text { Lack of Frozen } \\
\text { Requirements }\end{array}$ & $\mathrm{P}$ & $\begin{array}{l}\text { Team Members Lack Requisite } \\
\text { Knowledge and/or Skills }\end{array}$ & $\mathrm{P}$ \\
\hline 10 & Functionality Gaps & $\mathrm{T}$ & $\begin{array}{l}\text { Lack of People Skills in } \\
\text { Project Leadership }\end{array}$ & $\mathrm{P}$ & $\begin{array}{l}\text { Subject Matter Experts are } \\
\text { Overscheduled }\end{array}$ & $\mathrm{P}$ \\
\hline
\end{tabular}

T: Technisches Teilsystem, S: Soziales Teilsystem, P: Projektmanagement

\section{Diskussion der Ergebnisse}

Zwei verschiedene Begründungen könnten die Diskrepanz zwischen den Rangfolgen erklären: Zum einen könnte die Kontrollierbarkeit der Risikofaktoren eine Rolle bei der Risikobewertung spielen. Wie bisherige Studien zeigen, werden Projektmanager Risikofaktoren höher bewerten, wenn sie diese nur begrenzt kontrollieren können. Risikofaktoren, die aus Sicht des Projektmanagers nicht beeinflusst werden können, werden ausgeblendet und Risikofaktoren, die vollständig kontrolliert werden können, werden als unbedeutend eingeschätzt (March und Shapira 1987; Schmidt et al. 2001). Wir argumentieren, dass Risikofaktoren, die dem technischen Teilsystem zugeordnet werden können, aus Sicht des Projektmanagers nicht kontrollierbar sind, da diese Risikofaktoren bereits vor Projektstart, beispielsweise durch die bestehende IT-Architektur des Kunden, vorgegeben sind. Risikofaktoren des sozialen Teilsystems liegen bis zu einem gewissen Maß innerhalb des Kontrollbereichs des Projektmanagers, so wie bspw. die Beziehung zum Kunden und den zukünftigen Nutzern. Schließlich haben Projektmanager vollständige Kontrolle über Risikofaktoren innerhalb der Projektmanagementdomäne, so z. B. die Projektplanung und die Projektbesetzung.

Im direkten Unternehmensumfeld, wird die Bewertung der Risikofaktoren jedoch als organisationales und politisches Instrument benutzt. Daher kann die 
Bewertung durch die Projektmanager als mikropolitisch beeinflusst bezeichnet werden (Crozier und Friedberg 1980). Projektmanager nutzen den Risikomanagementprozess, um unkontrollierbare Risikofaktoren, die den Projekterfolg stark gefährden können, zu eskalieren. Desweiteren priorisieren sie Risikofaktoren herunter, die auf eigene Fähigkeiten oder die ihrer Arbeitskollegen zurückzuführen sind (Crozier und Friedberg 1980). Auf diese Weise führen mikropolitische Mechanismen dazu, dass die aus Unternehmensperspektive wichtigsten Risikofaktoren berichtet werden. Projektmanager wollen sich durch den Risikobericht von der Verantwortlichkeit für unkontrollierbare Risiken lossprechen und berichten diese daher mit höchster Priorisierung. Im Gegensatz dazu, werden Risikofaktoren aus den Domänen „Soziales Teilsystem“ oder „Projektmanagement" herunter priorisiert, um sich selbst oder Kollegen nicht in einem schlechten Licht dastehen zu lassen (Crozier und Friedberg 1980).

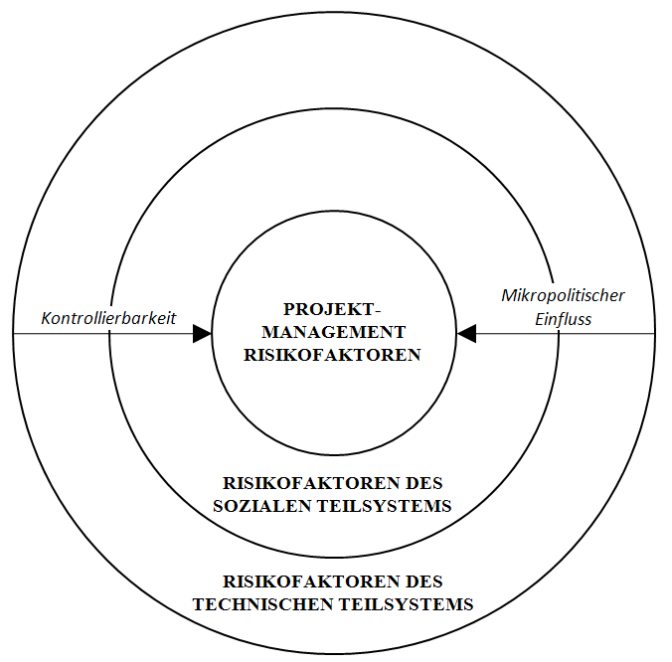

\section{Abbildung 1: Kontrollierbarkeit und Mikropolitischer Einfluss im Kontext der Risikodomänen von Wallace et al. (2004)}

Abbildung 1 zeigt ein Modell, das diese beiden Argumentationslinien miteinander verbindet. Das Ausmaß der Kontrolle erhöht sich ausgehend von der Domäne des technischen Teilsystems hin zu der Domäne "Projektmanagement". Ebenso verhält sich das Potenzial für mikropolitisch begründeten Einfluss.

Wie March und Shapira (1987) zeigen, fokussieren sich Manager generell auf Risiken, die sie als kontrollierbar einstufen. Daher besteht eine hohe Wahrscheinlichkeit, dass sich Projektmanager im Zuge einer Befragung oder eines Interviews auf kontrollierbare Risikofaktoren beschränken. Dieser Einfluss wird in manchen Studien durch die Fragestellung verstärkt. Bspw. baten Schmidt et al. (2001) die von Ihnen befragten Projektmanager, diejenigen Risikofaktoren zu identifizieren und zu bewerten, denen sie am meisten Ressourcen und 
Aufmerksamkeit widmen. Ein genauerer Blick auf die Priorisierungen von Schmidt et al. (2001) und Kappelman et al. (2006) verstärkt diese Überlegung: Die Risikofaktoren dieser Studien sind entweder direkt kontrollierbar, bspw. kann das Fehlen von Projektmanagementfähigkeiten durch Trainings oder ToolUnterstützung kompensiert werden, oder teilweise kontrollierbar, wie bspw. fehlende Managementunterstützung, die durch ständige Kommunikationsbemühungen beeinflusst werden kann.

Im Gegensatz dazu hebt der mikropolitische Einfluss, der in Projektrisikoberichten von ALPHA sichtbar wird, diejenigen Risikofaktoren hervor, die als durch den Projektmanager unkontrollierbar wahrgenommen werden und den Projekterfolg beträchtlich gefährden können. Wie zu sehen ist, enthält unsere Priorisierung unkontrollierbare Risikofaktoren des technischen Teilsystems, wie z. B. "Inadequate Technical Infrastructure", "Complex System Architecture" und "Development Dependencies". Diese Risikofaktoren sind auf Unternehmensebene (teilweise) kontrollierbar. So kann das Steuerungsgremium des Projekts Verträge nachverhandeln oder das Projekt im schlimmsten Fall abbrechen. Falls die technische Infrastruktur des Kunden zu komplex erscheint, kann das Projekt so lange verschoben werden, bis Altsysteme konsolidiert wurden. Derartige Entscheidungen liegen jedoch außerhalb des Einflussbereichs des Projektmanagers. Trotz der Tatsache, dass der Projektmanager diese Risikofaktoren nicht kontrollieren kann, dürfte eine Berichterstattung essentiell für den Projekterfolg sein und enthebt den Projektmanager zudem der Verantwortung, falls das Projekt fehlschlägt.

Analog dazu könnte ein mikropolitischer Einfluss ebenso die niedrigere Bedeutung der Risikofaktoren erklären, die der Projektmanagementdomäne zugeordnet werden können. Bspw. könnte sich ein Projektmanager Interessenskonflikten gegenübersehen, wenn er seine/ihre eigenen Fähigkeiten oder die von Vorgesetzten und Teammitgliedern bewertet. Das in dieser Hinsicht prominenteste Beispiel in den untersuchten Priorisierungen sind die mangelhaften Projektmanagementfähigkeiten. Während dieser Risikofaktor von Schmidt et al. (2001) an erste Stelle gesetzt wird, findet er sich in der Priorisierung der ALPHA Projektmanager nicht unter den zehn bedeutendsten Risiken. Andere Beispiele dieser Art stellen die Risikofaktoren „Lack of Top Management Support“ oder „Lack of Required Skills in Project Personnel“ dar.

Insgesamt bieten wir mit den beiden Dimensionen „Kontrollierbarkeit“ und „Mikropolitischer Einfluss“ erste Ansätze, die die zugrunde liegenden Mechanismen der Risikobewertung von Projektmanagern erklären könnten.

\subsection{Implikationen für die Forschung}

Frühere Studien stimmen größtenteils bzgl. der relativen Bedeutung von Risikofaktoren in IT-Projekten überein. Es scheint klar, dass Risikofaktoren, die dem technischen Teilsystem zugeordnet werden können, keine größere Gefahr für 
den Projekterfolg darstellen. Die Priorisierung der Projektmanager von ALPHA kontrastiert diese Ansicht: Fünf der zehn bedeutendsten Risikofaktoren lassen sich dem technischen Teilsystem zuordnen. Wir zeigen zwei mögliche Gründe für diesen Unterschied auf: Zum einen sind Risikofaktoren des sozialen Teilsystems und des Projektmanagements eher kontrollierbar und deshalb sichtbarer für Projektmanager, die an einer Umfrage teilnehmen oder interviewt werden. In der Praxis wirken zum anderen auch weitere Faktoren, wie beispielsweise mikropolitische Einflüsse auf die Bedeutung eines Risikofaktors ein. Aus diesem Grund erscheint es notwendig, Risikofaktoren als soziale Konstrukte zu betrachten. Je nach Kontext und Ursache der Bewertung ist es möglich, dass sich die Risikowahrnehmung verändert.

Desweiteren argumentieren wir, dass verschiedene Perspektiven auf ITProjektrisikofaktoren das Verständnis im Bereich Projektmanagement verbessern können. Viele der untersuchten Studien - die vorliegende eingeschlossen konzentrieren sich auf den Projektmanager als zentrales Untersuchungsobjekt. Die Miteinbeziehung weiterer Perspektiven, wie bspw. die der Teammitglieder, des Steuerungskomitees oder der Führungskräfte würde das Verständnis für ITProjektrisikofaktoren weiter erhöhen.

\subsection{Implikationen für die Praxis}

Obwohl sich unsere Forschung noch in einem frühen Stadium befindet, sehen wir verschiedene Implikationen für die Praxis. Zum einen können Projektmanager unsere Priorisierung der Risikofaktoren als Ergänzung der eigenen Checklisten verwenden und so sicherstellen, möglichst keine Risikofaktoren außer Acht zu lassen, die außerhalb der Kontrolle des Projektmanagers liegen. In dieser Hinsicht heben wir nicht nur die Bedeutung von Risikofaktoren des technischen Teilsystems heraus, sondern auch umgebungsbezogene Risikofaktoren, wie das Vertragsdesign ("Non-T\&M Payment Terms") oder die finanzielle Gesundheit des Kunden ("Customer Financial Obligations"). Zusätzlich zeigt unsere Priorisierung die Wichtigkeit von verschiedenen Rollen innerhalb des Risikomanagementprozesses, damit möglichst viele Risikofaktoren identifiziert werden können. Schließlich betont unsere Studie die Auswirkung von zusätzlichen Dimensionen, wie bspw. des mikropolitischen Einflusses, auf den Risikomanagementprozess. Projektrisikomanagement kann insgesamt nicht alleinige Aufgabe des Projektmanagers sein, sondern muss durch das Management, Steuerungsgremien und das unternehmensweite Risikomanagement unterstützt werden. 


\section{Schlussfolgerung und Ausblick}

In unserer Studie erstellen wir, basierend auf einem Archiv an Projektrisikodaten, eine Priorisierung von Risikofaktoren und vergleichen diese mit bestehenden Rangfolgen von Risikofaktoren bei IT-Projekten. Im Gegensatz zu früheren Studien, die personen- und prozessbezogene Risikofaktoren in den Vordergrund stellen, hebt unsere Analyse die Bedeutung von Risikofaktoren hervor, die dem technischen Teilsystem zugeordnet werden können. Wir schlagen vor, dass die Diskrepanz zwischen den beiden Rangfolgen aufgelöst werden kann, indem die Risikowahrnehmung aus der Sicht zweier Dimensionen untersucht wird: Kontrollierbarkeit und mikropolitischer Einfluss. Wir argumentieren zudem, dass die Diskrepanz auf verschiedene Perspektiven der Risikobedeutung zurückzuführen ist.

Obwohl die Ergebnisse einigen Einschränkungen hinsichtlich ihrer Generalisierungsfähigkeit unterliegen (z. B. lediglich Daten eines Unternehmens oder Auswahl der Variablen zur Bestimmung der Risikoexposition), schlagen wir eine neue Perspektive auf die relative Bedeutung von Risikofaktoren in IT-Projekten vor. Zudem stellen wir erste Erklärungsansätze dar, die sich mit den bestehenden Forschungsergebnissen vereinen lassen und helfen, ein besseres Verständnis von Risikowahrnehmung und Risikoverhalten von Projektmanagern zu entwickeln. Etwaige Limitationen sind dabei Gegenstand weiterer Forschungsarbeit.

Insgesamt erlauben unsere Ergebnisse so einen ersten Ansatz hinsichtlich des Verständnisses der relativen Bedeutung von Risikofaktoren in IT-Projekten. Wir werden uns in Zukunft darauf konzentrieren, die hier präsentierten Argumente zu festigen. Es scheint wahrscheinlich, dass mikropolitische Einflüsse die Bewertung von Risikofaktoren verändern. Nach unserem Wissen wurde dieser Einfluss bislang noch nicht in der Literatur aufgearbeitet. Weiterhin argumentieren wir, dass zusätzliche Domänen von Risikofaktoren, so wie der Vertrag, Steuerungsmodelle und der Kunde in die Risikopriorisierung mit einfließen sollte. Alles in allem trägt unsere Forschungsarbeit zur Entwicklung des Projektrisikomanagements bei, indem ein erstes Modell vorgeschlagen wird, das die Ergebnisse bestehender Studien miteinander verbindet und neue Ansätze liefert, die Mechanismen hinter der Risikowahrnehmung von IT-Projektmanagern zu verstehen.

\section{Literatur}

Alter S, Ginzberg M (1978) Managing Uncertainty in MIS Implementation. Sloan Management Review, Vol. 20 (1978): 23-31.

Barki H, Rivard S, Talbot J (1993) Toward an Assessment of Software

Development Risk. Journal of Management Information Systems, Vol. 10 (1993): 203-225.

Boehm B (1991) Software Risk Management: Principles and Practices. IEEE Software, Vol. 8 (1991): 32-41. 
Chapman C, Ward S (1996) Project risk management: processes, techniques and insights. John Wiley, Chichester.

Crozier M, Friedberg E (1980) Acotrs and systems: The politics of collective action. University of Chicago, Chicago.

Jiang J, Klein G (2000) Software development risks to project efectiveness. The Journal of Systems and Software, Vol. 52 (2000): 3-10.

Kappelman L, McKeeman R, Zhang L (2006) Early Warning Signs of IT Project Failure: The Dominant Dozen. IT Project Management, Vol. 23 (2006): 31-37.

March J, Shapira Z (1987) Managerial Perspectives on Risk and Risk Taking. Management Science, Vol. 33 (1987): 1404-1419.

McFarlan W (1981) Portfolio approach to information systems. Harvard Business Review, Vol. 59 (1981): 142-151.

Moynihan T (1997) How Experienced Project Managers Assess Risk. IEEE Software, Vol. 14 (1997): 35-41.

Schmidt R, Lyytinen K, Keil M, Cule P (2001) Identifying Software Project Risks: An International Delphi Study. Journal of Management Information Systems, Vol. 17 (2001): 5-36.

Tiwana A, Keil M (2004) The One-Minute Risk Assessment Tool.

Communications of the ACM, Vol. 47 (2004): 73-78.

Wallace L, Keil M, Rai A (2004) How Software Project Risk Affects Project Performance: An Investigation of the Dimensions of Risk and an Exploratory Model. Decision Sciences, Vol. 35 (2004): 289-321.

Zmud R (1980) Management of Large Software Development Efforts. MIS Quarterly, Vol. 4 (1980): 45-56. 